\title{
The effect of New Zealand blackcurrant extract on recovery following eccentric exercise induced muscle damage
}

\author{
M. Coelho, S. Buxton, R. Butcher, D. Foran, R. Manders and J. Hunt \\ Faculty of Health and Medical Sciences, University of Surrey, Guildford, UK.
}

Exercise induced muscle damage (EIMD) is characterized by a decrease in muscular strength and range of motion (ROM), localised inflammation and muscle soreness, and an increase in serum concentration of intramuscular proteins, such as creatine kinase $(\mathrm{CK})^{(1)}$. The consumption of blackcurrants, rich in anthocyanins, may improve recovery following strenuous exercise as a result of their antioxidant and anti-inflammatory properties ${ }^{(2)}$. We examined the effect of New Zealand blackcurrant (NZBC) extract on indices of muscle damage and inflammation (interleukin-6, IL-6) following strenuous eccentric exercise.

Using a double-blind, placebo-controlled, parallel design, 13 healthy females consumed either NZBC extract $(n=6)$ or placebo $(n=7)$ capsules for 7 days prior to, on the day of, and 4 days following EIMD induced by 60 maximal eccentric contractions of the bicep brachii. Elbow flexion maximal voluntary contraction (MVC), perceived muscle soreness, ROM, mid-arm circumference (MAC), serum CK and plasma IL-6, were measured at baseline, and 24, 48, 72 and 96 hours post EIMD. An additional measurement for MVC was taken immediately post EIMD.

No significant differences were found between groups at baseline. A significant decrease in MVC was observed immediately following the EIMD protocol in both groups $(p=0.006)$. MVC returned to baseline values at 96 hour post EIMD $(p=0.097)$ with no difference in the rate of recovery between groups. Perceived muscle soreness was attenuated in the NZBC vs. placebo group at $24(p=0 \cdot 038)$ and 48 hours $(p=0.031)$ (Figure 1). Serum CK displayed a similar trend at $72(p=0.061)$ and 96 hours $(p=0.062)$ but no statistical difference between groups was found (Figure 2). No differences between groups in ROM, MAC and IL-6 were detected during the recovery period.

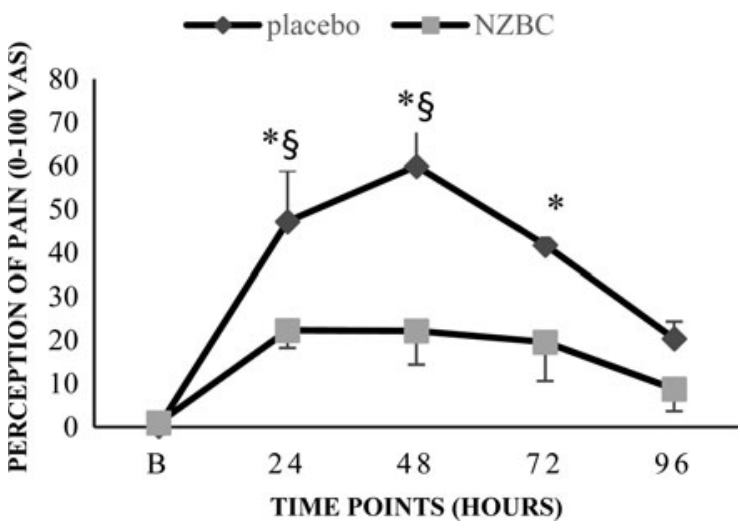

Fig. 1. Perception of Pain.

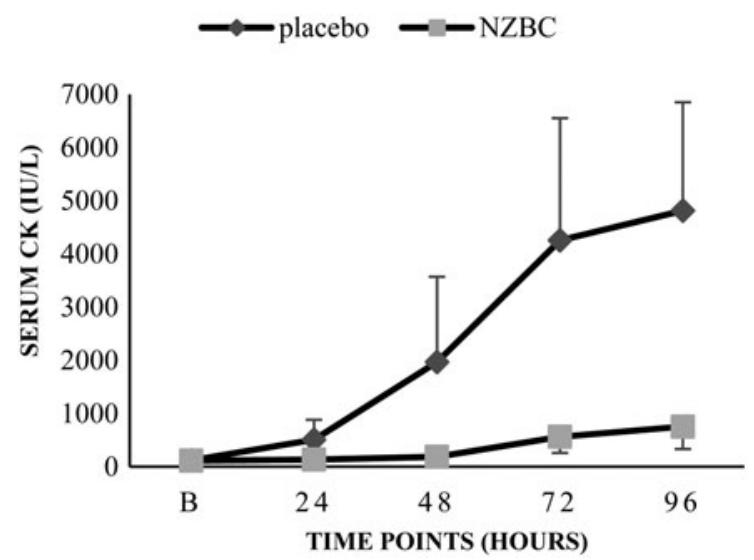

Fig. 2. Serum CK.

The preliminary data from this ongoing study provides evidence that the consumption of a NZBC extract reduces muscle soreness and may attenuate muscle damage following strenuous eccentric exercise, although mechanisms have not been clarifed. These findings are of practical importance in recreationally active and athletic populations, who may benefit from accelerated recovery following EIMD.

The supplement utilised in this study was provided by CurraNZTM (Health Currancy Ltd.)

1. Armstrong RB (1990) Initial events in exercise-induced muscular injury. Medicine and science in sports and exercise 22(4), 429-35.

2. Li D, Wang P, Luo Y, Zhao M et al. (2015) Health benefits of anthocyanins and molecular mechanisms: update from recent decade. Crit. Rev. Food. Sci. Nutr. doi:10.1080/10408398.2015.1030064. 